\title{
The Current Status of Renal Transplant Recipients with Hepatitis C: A Single Center Experience
}

\author{
Koichi Kozaki ${ }^{1,2}$, Naoki Adachi ${ }^{1}$, Akiho Okada ${ }^{1}$, Taketo Kato ${ }^{1}$, Kenji Yuzawa ${ }^{2}$, Haruo Ohtani ${ }^{3,4}$ and \\ Tohru Terashima ${ }^{1}$ \\ ${ }^{1}$ Department of Surgery, National Hospital Organization, Mito Medical Center, Ibaraki, Japan \\ ${ }^{2}$ Department of Transplantation Surgery, National Hospital Organization, Mito Medical Center, Ibaraki, Japan \\ ${ }^{3}$ Department of Pathology, National Hospital Organization, Mito Medical Center, Ibaraki, Japan \\ ${ }^{4}$ Department of Pathology, Mito Saiseikai General Hospital, Ibaraki, Japan
}

Corresponding author: Koichi Kozaki, Department of Surgery and Transplantation Surgery, National Hospital Organization, Mito Medical Center, Ibaraki, Japan, Tel: 81-29-240-7711; Fax: 81-29-240-7788; E-mail: k.kozaki.d@mn.hosp.go.jp

Received: 31 January 2017; Accepted: 03 March 2017; Published: 06 March 2017

Citation: Kozaki K, Adachi N, Yuzawa K, et al. The Current Status of Renal Transplant Recipients with Hepatitis C: A Single Center Experience. Ann Clin Lab Res. 2017, 5: 1.

\section{Abstract}

Renal transplantation (RTx) may result in the development of liver disorder/hepatic cancer or hepatitis C virus (HCV)related membranoproliferative glomerulonephritis, which may lead to graft loss, in renal failure patients with previously untreated HCV infection. Long-term survival is longer in HCV antibody-positive renal transplant recipients than in HCV antibody-negative/-positive dialysis patients, whereas renal graft survival and patient survival are lower in HCV antibody-positive renal transplant recipients than in $\mathrm{HCV}$ antibody-negative patients; thus, HCV treatment should be administered to HCV antibodypositive renal transplant recipients. Interferon/ribavirin (IFN/Rib) therapy is one of the current HCV treatments in Japan. In renal failure patients including those undergoing hemodialysis, Rib is contraindicated because of possible kidney function problems; IFN monotherapy is indicated for these patients. However, in clinical practice, IFN monotherapy is not performed adequately because of efficacy problems or adverse reactions to IFN, leading to the presence of many $\mathrm{HCV}$ carriers and patients with previous HCV infection among hemodialysis patients in the RTx waiting list. The sustained virological response in patients receiving IFN therapy after RTx is low. Many patients discontinue the therapy and some develop graft loss because of IFN-related acute rejection. Therefore, dialysis patients with $\mathrm{HCV}$ infection who are scheduled for RTx should first receive IFN therapy. In Japan, daclatasvir/ asunaprevir combination therapy was introduced as an IFN-free antiviral therapy in September 2015. However, its safety in dialysis patients has not been established, and these patients still require IFN therapy. We report the current state of renal transplant recipients with $\mathrm{HCV}$ at our hospital.

Keywords: Renal transplant; Hepatitis C; Hemodialysis

\section{Introduction}

The number of dialysis patients in Japan has been increasing by approximately 10,000 annually, and the total number was $>300,000$ at the end of 2016 [1]. Among these patients, the proportion of hemodialysis (HD) patients with hepatitis $\mathrm{C}$ virus (HCV) infection is still high (approximately 10\%) [2]. HD patients with $\mathrm{HCV}$ infection have poorer prognosis than HD patients with no HCV infection; if possible, it is preferable for patients with renal failure to have clearance of HCV virus. Interferon (IFN)/ribavirin (Rib) therapy is one of the current treatments for HCV $[3,4]$; however, Rib is contraindicated in patients with renal failure, including those receiving HD, because of possible kidney function problems. IFN monotherapy is indicated in these patients $[5,6]$. In the clinical practice of dialysis treatment in Japan, IFN monotherapy is not performed adequately because of problems with its efficacy or adverse reactions to IFN, and this result in the presence of many HCV carriers and persons previously infected with HCV among HD patients who are on the RTx waiting list. Moreover, some patients develop liver disorder/hepatic cancer due to HCV or HCV-related membranoproliferative glomerulonephritis (MPGN) after renal transplantation (RTX), and experience graft loss [2,7-9]. After RTx, it is recommended that IFN/Rib therapy be administered to these patients according to their renal function. We report the current state of renal transplant recipients with HCV at our hospital and that of patients who received antiviral therapy for HCV after RTx. As there were patients with an estimated glomerular filtration rate $(\mathrm{eGFR})$ of $<50\left(\mathrm{~mL} \cdot \mathrm{min}^{-1} \cdot 1.73 \mathrm{~m}^{-2}\right)$ after $\mathrm{RTx}$, the use of Rib in patients after RTx was performed with the approval of the ethics review committee of our hospital.

\section{Patients}

We conducted this retrospective observational study to examine patients who were performed RTx at Mito Medical Center from May 2006 to December 2016. Of the 87 patients 
who underwent RTx who were 69 living-donor RTx and 18 cadaveric RTx, 9 patients (4 with living-donor RTx, 5 with cadaveric RTx) were HCV-patients. These 9 patients were all men with the mean age of 55.8 (49-80) years, mean duration of dialysis treatment of 227.7 (1-384) months, and mean follow-up time of 1947.5 (849-2.761) days (Table 1).

Table 1 Clinical characteristics (2006.5 2016.12).

\begin{tabular}{|l|l|l|l|}
\hline & HCV & Non-HCV & $\begin{array}{l}\text { p- } \\
\text { value }\end{array}$ \\
\hline Number of case & 9 & 78 & - \\
\hline Men & 9 & 51 & - \\
\hline Women & 0 & 27 & - \\
\hline Mean recipient age (years old) & 55.8 & 43.9 & 0.008 \\
\hline
\end{tabular}

Frequency of renal transplantation

\begin{tabular}{|l|l|l|l|}
\hline 1st & 6 & 72 & - \\
\hline 2nd & 3 & 5 & - \\
\hline 3rd & 0 & 1 & - \\
\hline
\end{tabular}

Kind of renal transplantation

\begin{tabular}{|l|l|l|l|}
\hline Living & 4 & 65 & - \\
\hline Cadaver & 5 & 13 & - \\
\hline Mean duration of dialysis (month) & 227.7 & 84 & 0.02 \\
\hline Mean follow-up duration (month) & 162.3 & 161.1 & N.S \\
\hline
\end{tabular}

Underlying nephropathy

\begin{tabular}{|l|c|c|c|}
\hline Chronic glomerulonephritis & 6 & 34 & - \\
\hline IgA nephropathy & 0 & 13 & - \\
\hline Diabetic nephropathy & 1 & 7 & - \\
\hline Cystic kidney & 2 & 1 & - \\
\hline Others & 0 & 23 & -
\end{tabular}

\section{Limitation}

In late years RTx of our country is approximately 1,500 cases a year. In addition, it is the present status in our area that there are extremely few RTx with approximately 5-10 cases a year. The patients in this study became the small group from these reasons.

\section{Methods}

We collected data for patient age, gender, primary diagnosis of renal disease (underlying nephropathy), frequency and kind of RTx, duration of dialysis, graft and patient survival. We evaluated laboratory data (Hemoglobin; Hb, Hematocrit; $\mathrm{Ht}$, aspartate aminotransaminase; AST, alanine aminotransferase; ALT, serum creatinine; $\mathrm{S}-\mathrm{Cr}$ ) and calculated estimated glomerular filtration rate (eGFR). All data were collected from medical records to perform this retrospective study.
IFN/Rib combination therapy was given to two HCV-patients with high viral load who also had severe proteinuria, liver disorder, and MPGN diagnosed with renal graft biopsy. In IFN/Rib therapy, polyethylene glycol-IFN $\alpha-2 a$ (90 $\mu \mathrm{g} /$ week) and Rib (200-400 mg/day) were administered. Rib blood concentration level was periodically measured, and the Rib dose was adjusted at the levels necessary to reach its effective concentration in the blood $(2500 \mathrm{ng} / \mathrm{mL})$, according to the seriousness of adverse reactions. Two patients of HCV group received IFN/Rib under our Ethical Review Board approval.

\section{Statistical Analysis}

Results are given as means value. Paired t-test was used for comparison of HCV and non-HCV patients. All analyses were performed as two-tailed; P-values of $<0.05$ were considered as statistically significant. StatView for Macintosh (version 5.0 Abacus Corporation, Baltimore, MD, USA) was used for statistical analysis.

\section{Results}

The laboratory test results of renal graft and liver function at present are as follows:

mean $\mathrm{Hb} ; 11.7$ (9.2-13.8) g/dl, Ht; 36.8 (29.6-43.2) \%, AST; 20.1 (11-48) IU/L, ALT; 21.3 (7-37) IU/L S-Cr; 1.72 (0.74-3.53) $\mathrm{mg} / \mathrm{dL}$, eGFR; $38.1(21.7-43.5) \mathrm{mL} \cdot \mathrm{min}^{-1} \cdot 1.73 \mathrm{~m}^{-2}$. The comparison between the HCV and the non-HCV patient groups revealed no statistically significant difference in the $\mathrm{Hb}, \mathrm{Ht}$, AST, ALT S-Cr, and eGFR during the observation period. The renal graft survival rate was significantly lower in the HCV group than in the non-HCV group ( $44.4 \%$ vs. $80.8 \%, p=0.03$ ) but patient survival rate was no statistically significant difference in both groups (Table 2).

Table 2 Comparison of HCV and non-HCV patient's clinical results.

\begin{tabular}{|l|l|l|l|}
\hline Parameters & HCV & Non-HCV & p-value \\
\hline Mean Hemoglobin (g/dL) & 11.7 & 12.6 & N.S \\
\hline Mean Hematocrit (\%) & 36.8 & 38.4 & N.S \\
\hline Mean AST (IU/L) & 20.1 & 18.3 & N.S \\
\hline Mean ALT (IU/L) & 21.3 & 13.6 & N.S \\
\hline Mean serum creatinine (mg/dL) & 1.72 & 1.49 & N.S \\
\hline $\begin{array}{l}\text { Mean eGFR (mL·min-1·1.73 } \\
\text { m-2) }\end{array}$ & 38.1 & 43 & N.S \\
\hline $\begin{array}{l}\text { Renal graft survival } \\
\text { Patinet survival }\end{array}$ & $4(44.4 \%)$ & $63(80.8 \%)$ & 0.03 \\
\hline
\end{tabular}

The results of the TaqMan HCV-RNA assay were $5.6 \pm 2.4$ (log) before RTx and $5.5 \pm 2.8(\mathrm{log})$ in the latest examination, showing no significant changes in the viral load, which remained high, in the course of RTx $(p=0.95)$. Six of nine patients developed liver disorder after RTx; liver-supporting therapy was performed on all these patients. Of the 6 patients, 
2 received IFN/Rib therapy because of severe proteinuria, liver disorder, and MPGN diagnosed with renal graft biopsy. Anemia was noted in both patients. However, the 2 patients continued the therapy, receiving periodic measurements of Rib levels in the blood, although erythropoietin preparations were used and the use of Rib was suspended as appropriate. Sustained virological response (SVR) was achieved in one patient; however, hemodialysis was restarted in both patients because of impaired renal graft function due to MPGN.

\section{Discussion}

One of the above-described two patients, a 43-year-old man, was infected with HCV at age 20 years from a transfusion required after a traffic accident. He started dialysis for diabetic nephropathy at age 40 years. In April 2007, this patient with a previously untreated HCV underwent living-donor RTx (donor: his 41-year-old wife). Around January 2012, proteinuria (>1 g/ day) developed; renal biopsy was performed, establishing the diagnosis of MPGN (Figure 1). Anti-HLA antibodies also developed coincidentally, we also suspected antibodies induced rejection, double filtration plasmapheresis (DFPP) plus steroid bolus therapy were performed. Proteinuria ( $>5 \mathrm{~g} /$ day), liver disorder, and the TaqMan HCV test detected HCV-RNA quantities $\geq 7.8$ (log). Virus removal and eradication by DFPP plus IFN/Rib therapy was performed. Consequently, the HCVRNA levels in the TaqMan test decreased to the lower detection limit or below, in May 2013. Anemia as an adverse reaction of IFN/Rib therapy developed in the patient. However, he continued the therapy, receiving periodic measurements of Rib levels in the blood, by using erythropoietin preparations or by suspending the use of Rib as appropriate, and thereby achieving SVR (Figures 2 and 3).

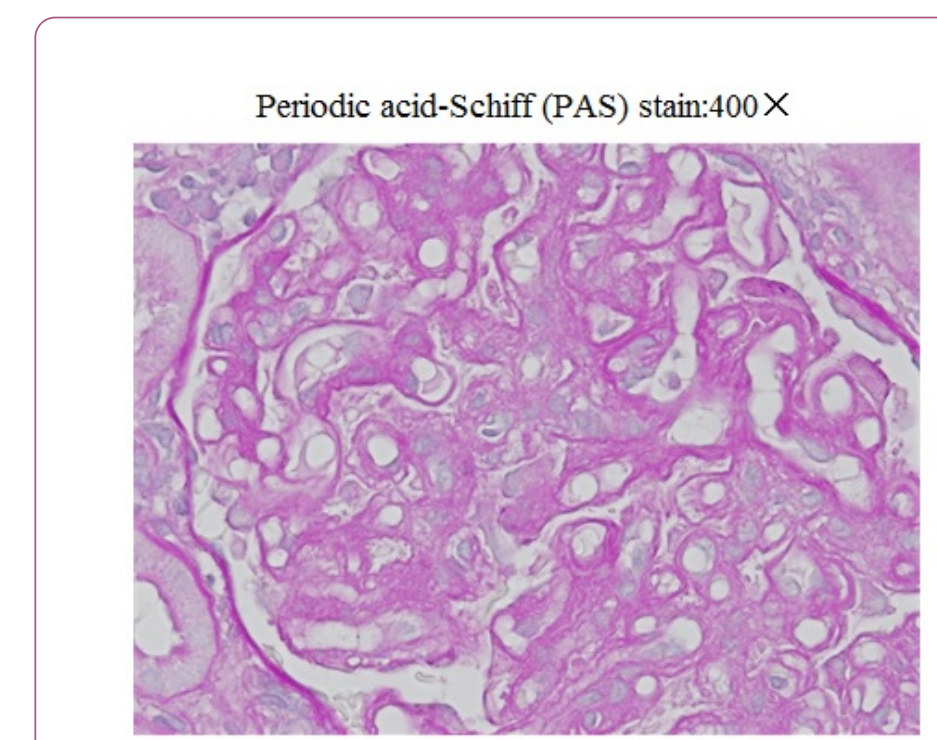

\section{Immunofluorescence: $400 \times \operatorname{IgG} \operatorname{IgM} \operatorname{IgA} C 3 c \mathrm{C} 4 \mathrm{~d}(+)$}

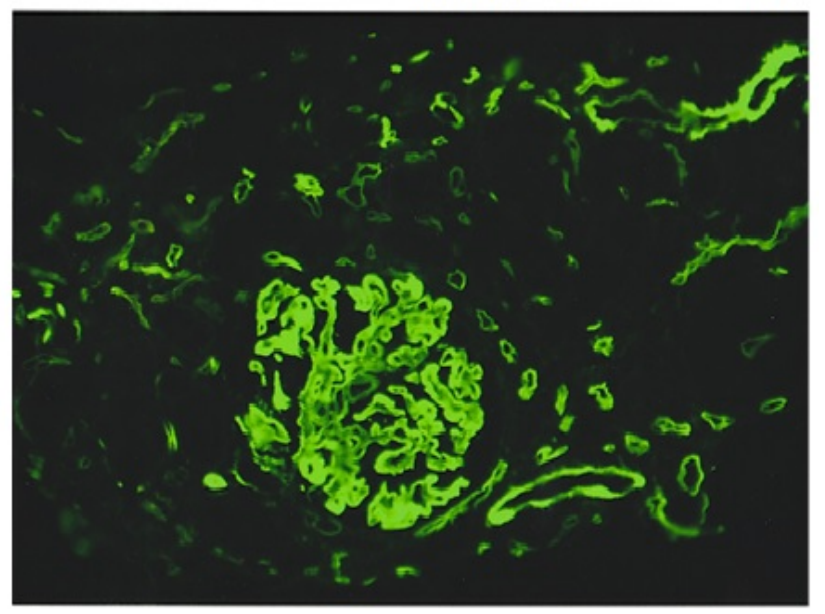

Transplant renal biopsy:

1) Proliferation of mesangial and endothelial cells and expansion of the mesangial matrix

2) Mesangial interposition into the capillary wall, giving rise to a double-contour of basement membrane

3) Immunofluorescence; deposition of various immunoglobulin $\rightarrow$ Membranoproliferative glomerulonephritis (MPGN)

Figure 1 Pathological findings of transplant renal biopsy.

\section{Conclusion}

All our HCV-patients had eGFR $\leq 50 \mathrm{~mL} \cdot \mathrm{min}^{-1} \cdot 1.73 \mathrm{~m}^{-2}$. In these patients, Rib is contraindicated, and, in principle, IFN monotherapy is indicated. While there are reports of IFN therapy exerting relatively high effects on HCV in RTx patients, there are also reports of IFN therapy resulting in renal graft loss due to IFN-induced rejection; careful measures must be taken for these patients. IFN/Rib therapy is considered to be effective in patients with HCV-related nephropathy (MPGN), and is currently an essential therapy for the maintenance of hepatic function and the long-term renal graft survival in HCVpatients receiving $\operatorname{RTx}[4,10,11]$. In Japan, daclatasvir/ asunaprevir (DCV/ASV) combination therapy was introduced as an IFN-free antiviral therapy in September 2015 [12]. The safety of this therapy in RTx patients or HD patients has not been established. If the efficacy and safety of DCV/ASV therapy in RTx patients and HD patients were to be verified in the future, IFN therapy will probably no longer be used. However, IFN therapy, which is unrelated to drug resistance, may be given to patients infected with multiple-drug resistant viruses arising from treatment with oral medications only. In Japan, IFN therapy should be used until the safety of DCV/ASV therapy is established, and IFN/Rib therapy must be considered for RTx recipients with $\mathrm{HCV}$ in certain circumstances. IFN/Rib can be given to RTx recipients with HCV 
if these patients undergo periodic blood tests for Rib levels and dose adjustments of Rib according to the evaluation of its efficacy/adverse reactions.

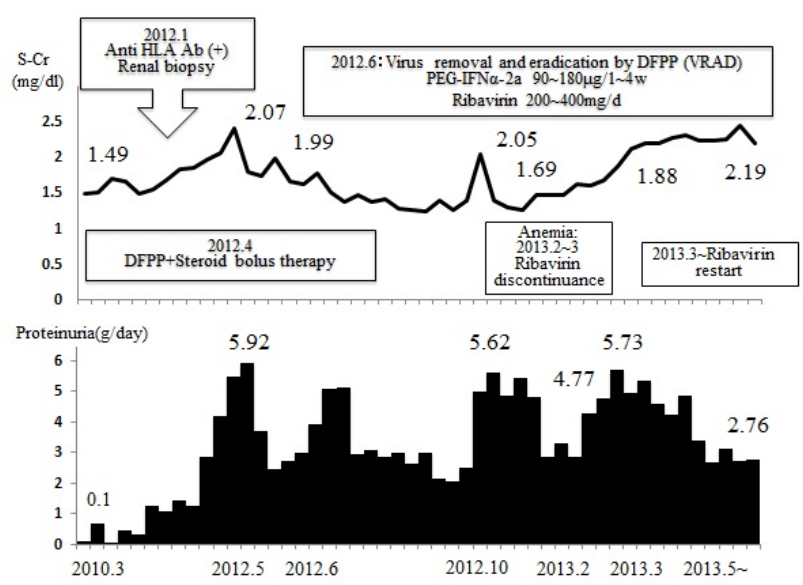

Figure 2 Clinical course-1.

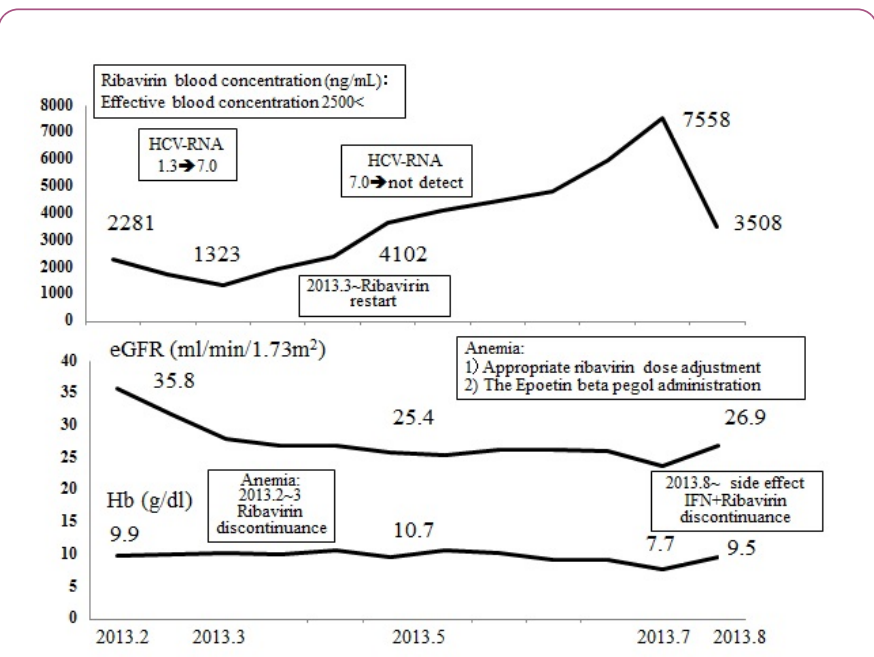

Figure 3 Clinical course-2.

In the future, HCV eradication therapy should be performed more actively in HCV-infected renal transplant recipients as a way to help protect and preserve their hepatic functions and achieve long-term survival of renal grafts.

In present, a transplant coordinator strongly pressures a dialysis facilities to work on it to have the HCV patients who undergo renal transplant receive treatment for the HCV before transplant. However, the HCV treatment to a patient on dialysis is not still provided enough in the dialysis facilities, and this point is a future problem.

\section{References}

1. Renal Data Registry (2016) Japanese Society for Dialysis Therapy.

2. Tabata H, Kikuchi K, Ishida H, Tanabe K, Nitta K (2012) Impact of hepatitis $C$ virus on living-donor kidney transplantation. J Jpn Soc Dial Ther 45: 1147-1153.

3. Kuboki $M$, lino $S$, Okuno $T$, Omata $M$, Kiyosawa $K$, et al. (2007) Peginterferon alpha-2a (40 KD) plus ribavirin for the treatment of chronic hepatitis $C$ in Japanese patients. J Gastroenterol Hepatol 22: 645-652.

4. Dalrymple LS, Koepsell T, Sampson J, Louie T, Dominitz JA, et al. (2007) Hepatitis C virus infection and the prevalence of renal sufficiency. Clin J Am Soc Nephrol 2: 715-721.

5. Fabrizi F, Takkouche B, Lunghi G, Dixit V, Messa P, et al. (2007) The impact of hepatitis $C$ virus infection on survival in dialysis patients: J Viral Hepat 14: 697-703.

6. Gordon CE, Uhlig K, Lau J, Schmid CH, Levey AS, et al. (2008) Interferon treatment in hemodialysis patients with chronic hepatitis $C$ virus infection: a systematic review of the literature and meta-analysis of treatment efficacy and harms. Am J Kidney Dis 51: 263-277.

7. Cruzado JM, Casanovas-Taitavull, Torras J (2003) Pretransplant interfereon prevents hepatitis $C$ virus-associated glomerulonephritis in renal allografts by HCV-RNA clearance. Am J Transplant 3: 357-360.

8. Mahmoud IM, Sobh MA, El-Habashi AF, Sally ST, El-Baz M, et al. (2005) Interferon therapy in hemodialysis patients with chronic hepatitis C: study of tolerance, efficacy and post-transplantation course. Nephron Clin Pract 100: 133-139.

9. Yamabe $\mathrm{H}$, Johnson RJ, Gretch DR, Fukushi $\mathrm{K}$, Osawa $\mathrm{H}$, et al. (1995) Hepatitis C virus infection and membranoproliferative glomerulonephritis in Japan. J Am Soc Nephrol 6: 220-223.

10. Fabrizi F, Martin P, Dixit V, Bunnapradist S, Kanwal F, et al. (2005) Post-transplant diabetes mellitus and HCV seropositive status after renal transplantation: meta-analysis of clinical studies. Am J Transplant 5: 2433-2440.

11. The Japan Society of Hepatology (2016) Guidelines of hepatitis C treatment.

12. Kumada H, Suzuki Y, Ikeda K, Toyota J, Karino Y, et al. (2014) Daclatasvir plus asunaprevir for chronic HCV genotype $1 \mathrm{~b}$ infection. Hepatology 59: 2083-2091. 